\title{
Online Systems for Training the Evaluation of Deviations of Geometrical Characteristics
}

\author{
http://dx.doi.org/10.3991/ijoe.v9iS8.3355 \\ ${ }^{1,2}$ Teresa Restivo, ${ }^{2}$ José Rodrigues, ${ }^{1,2}$ Fátima Chouzal, ${ }^{3}$ Paulo Menezes, ${ }^{2}$ José Almacinha \\ ${ }^{1}$ IDMEC-Pólo FEUP, ${ }^{2}$ Universidade do Porto, Porto, Portugal, ${ }^{3}$ Universidade de Coimbra, Coimbra, Portugal
}

\begin{abstract}
In mechanical design, product manufacturing and product maintenance, geometrical characteristics such as straightness, flatness, roundness and cylindricity are very important, first in detail design with the specification of their tolerances in order to translate functional requirements into geometrical requirements and later, in the verification of the manufactured workpieces (measurement procedure) for compliance with the design objective. Coordinate Measuring Machines are very expensive equipment widely used to perform automated measurement, to inspect and to analyse engineering components in order to check their conformity with the specifications. This work presents augmented reality systems for conveying these concepts and to provide an introduction to the straightness and flatness evaluations. These systems intend to enable the users to explore the related concepts for training or educational purposes in a more immersive way.
\end{abstract}

Index Terms-Augmented reality, metrology, surface morphology, virtual reality.

\section{INTRODUCTION}

Laboratory classes enable the students to see by themselves how things work and experiment the effects of modifying some variables, inputs, etc. Furthermore, some technical tasks require practice that can only be acquired with hands-on experimentation. Thanks to the use of digital computers, it has been possible to create simulators that show on the computer screen the behaviour of many different experiments, some of which could never have been tested in reality, for most people. These simulators enable us to learn what to do, but not how to react. Virtual and augmented reality based simulators can bring the experience to a higher level. With them, the user can explore details of the experiment, having the feeling that $\mathrm{s} / \mathrm{he}$ is in the "presence" of the "real thing". Presence feeling and immersion are two of the most important added values that Augmented Reality (AR) and Virtual Reality (VR) can bring to any educational or training system. In fact, feeling that "I was there" and "I've sensed it" are two important ingredients for creating "unforgettable experiences" [1].

This work presents AR applications developed for training the evaluation of geometrical characteristics deviations.

"Geometrical product specification" and "Geometric dimensioning and tolerancing" are technical and standard terms used in engineering detail drawings to accurately describe parts. In mechanical design, product manufacturing and product maintenance, geometrical characteristics such as straightness, flatness, roundness and cylindricity are very important, first, in detail design, with the specification of their tolerances, in order to translate functional requirements into geometrical requirements and later, in the verification of the manufactured workpieces (measurement procedure) for compliance with the design goal [2]. For example, in machine tool guiding systems, the straightness verification is fundamental to guarantee the conformance of produced parts with their tolerance specifications [3].

\section{Straightness And Flatness ConcePts}

\section{A. Straightness}

By definition straightness is a property of a straight line. It characterizes a line (a line on a flat surface, a generating line on a cylindrical surface or a median line of a cylinder) in what concerns its deviation from an ideal straight line, but it is not sufficient for complete surface characterization.

The straightness of a single toleranced feature is deemed to be corrected when the feature is confined between two parallel straight lines and the distance between both is equal to or less than the value of the specified tolerance. The orientation of the straight lines shall be chosen so that the maximum distance between them is the least possible value. According to the ISO12780-1, the least squares reference line method provides a good approximation for the straightness deviation, although overestimating it.

Considering an extracted line $L \mathrm{~m}$ containing the surface points $\left(x_{\mathrm{i}}, y_{\mathrm{i}}\right)$ in the selected direction, we must obtain the least squares reference line (LSLI) as a line such that the sum of the squares of the local straightness deviations is a minimum, Fig. 1. The local straightness deviation, $a_{i}$, of each point $\left(x_{\mathrm{i}}, y_{\mathrm{i}}\right)$ from the least squares reference line (measured normally to it) can be calculated using (1), where $m$ is the LSLI slope and $b$ its $y$-axis intercept.

$$
a_{\mathrm{i}}=\frac{y_{\mathrm{i}}-m x_{\mathrm{i}}-b}{\sqrt{1+m^{2}}}
$$

The straightness deviation of the considered feature is the peak-to-valley straightness deviation (STRt) given by the value of the largest positive local straightness deviation, $a_{1}$, added to the absolute value of the largest negative local straightness deviation, $\left|a_{2}\right|$.

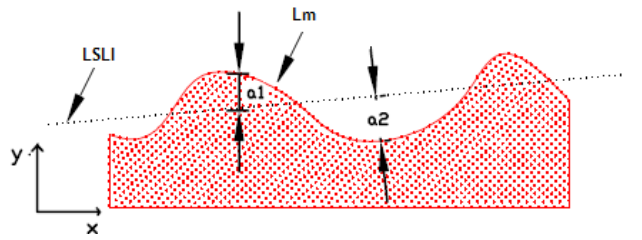

Figure 1. Least squares reference line method [ISO 12780-1]. 


\section{B. Flatness}

By definition flatness is a property of a plane. It characterizes a surface in what concerns its deviation from an ideal flat surface. Basically, its tolerance is used to control flat surfaces and, often, to qualify a surface as a primary datum.

The flatness of a single toleranced feature is deemed to be corrected when the feature is confined between two parallel planes and the distance between them is equal to or less than the value of the specified tolerance. The orientation of the planes shall be chosen so that the maximum distance between them is the least possible value. According to ISO 12781-1, the least squares reference plane method provides a good approximation for the flatness deviation, although overestimating it.

In order to obtain a reliable assessment of flatness, an appropriate extraction strategy for obtaining a representative set of points on the workpiece is required. In practice, it is often difficult to achieve a complete covering of the flatness feature. In these situations, more limited extraction strategies are employed. In general, the rectangular grid extraction strategy, consisting of equally spaced straightness profiles in two orthogonal directions to form a grid, is recommended as the sampling strategy for the assessment of the total feature of flatness [ISO 12781-2].

Considering the $n$ surface points $\left(X_{\mathrm{i}}, Y_{\mathrm{i}}, Z_{\mathrm{i}}\right)$ extracted along the lines $L \mathrm{~m}_{\mathrm{j}}$ established in the two selected orthogonal directions, we must obtain the least squares reference plane (LSPL) as a plane such that the sum of the squares of the local flatness deviations is a minimum, Fig.2.

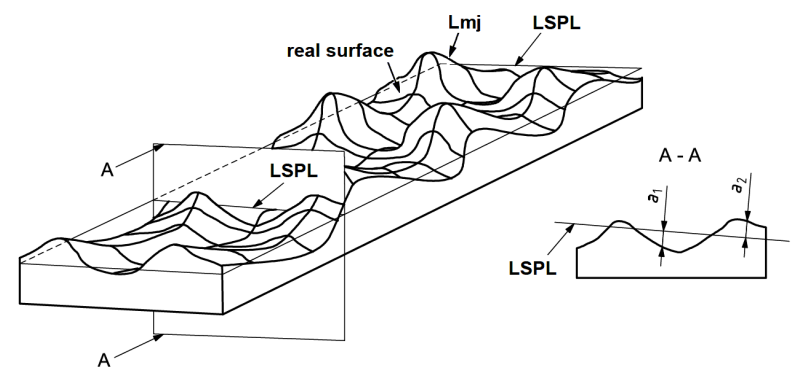

Figure 2. Least squares reference plane method [ISO 12781-1].

The least squares reference plane, (with an equation of the form $z=a x+b y$ ), passes through a point $\mathrm{O}$ with coordinates $\bar{X}=\sum X_{\mathrm{i}} / n ; \quad \bar{Y}=\sum Y_{\mathrm{i}} / n ; \quad \bar{Z}=\sum Z_{\mathrm{i}} / n$ that is taken as the origin of the new coordinates $x_{\mathrm{i}}=X_{\mathrm{i}}-\bar{X}$; $y_{\mathrm{i}}=Y_{\mathrm{i}}-\bar{Y}$ and $z_{\mathrm{i}}=Z_{\mathrm{i}}-\bar{Z}$.

The local flatness deviation, $a_{\mathrm{i}}$, of each point $\left(x_{i}, y_{i}, z_{i}\right)$ from the least squares reference plane (measured normally to it) can be calculated using (2), where $\hat{a}$ and $\hat{b}$ are the estimated coefficients of the least squares reference plane equation.

$$
a_{\mathrm{i}}=\frac{z_{\mathrm{i}}-\hat{a} x_{\mathrm{i}}-\hat{b} y_{\mathrm{i}}}{\sqrt{\left(\hat{a}^{2}+\hat{b}^{2}+1\right)}}
$$

The flatness deviation of the considered feature is the peak-to-valley flatness deviation (FLTt) given by the value of the largest positive local flatness deviation, $a_{1}$, added to the absolute value of the largest negative local flatness deviation, $\left|a_{2}\right|$.

\section{AUGMENTED REALITY SYSTEM FOR \\ STRAIGHTNESS AND FLATNESS EVALUATION}

\section{A. The concept}

Typical systems for straightness and flatness evaluation are very expensive and so they are not affordable in training and learning environments.

A low cost experimental set-up was built to evaluate and convey the straightness concept and is currently remotely available. However, using it for flatness evaluation would require a considerable investment. So, virtual systems based in the remote set-up were developed for straightness and flatness evaluation aiming to offer to the user online solutions with a god level of realism and immersion, and easily usable simultaneously by multiple users.

Taking advantage of augmented reality techniques and interaction capabilities by using colour tracking methods the applications can even surpass some of the remote experiment functionalities. Any user can make a different approach to the process, instead of a step-by-step guided process as that available online. And users can even handle the systems!

\section{B. The implemented AR systems}

The remote system uses a linear moving system positioned by a step motor and measurements are made by a digital-indicator gauge, as a low cost solution compared with standard solutions of optical type. The results (stepmotor position $v s$. measured coordinate) can be sent to the user email allowing later evaluation.

The AR applications are replicas of this system offering similar solutions. AR systems use, like in the real system, a linear moving system for the "measurement" procedure. And they also can email to the user the result file. For the flatness measurement the systems moves the measuring table, in which the specimen surface is placed, in a predefined way.

The AR applications use the facilities provided by the new HTML5 libraries that, in this case, access the webcam without the need for any plug-ins installed on the client side. The augmented reality was possible to be implemented using WebGL. For that purpose a library in JavaScript was used to make the implementation of WebGL easier. For the detection of the augmented reality markers and for colour tracking JavaScript libraries were employed. Bright yellow is the colour used for tracking.

The 3D models developed were made using Blender3D and exported in a format supported by our library [4].

\section{How to use}

As in any augmented reality application, a webcam is required to use the application. It needs to be run in Google Chrome to support the video capture without plugins. Available in the web page (http://paginas.fe.up.pt/ expat/experimentaportugal/labora torios.php?lang=pt), "laboratories/others".

The user should slide the horizontal yellow bar, named threshold, to a value where the marker is clearly seen in the black and white image. 


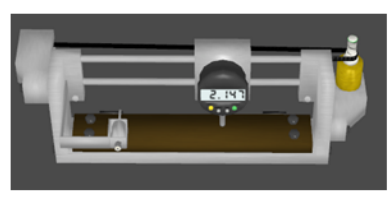

a)

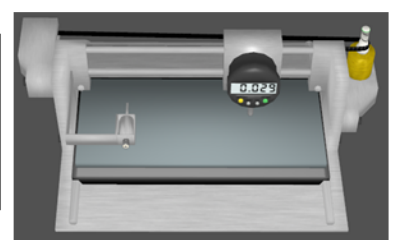

b)

Figure 3. Augmented reality system for a) straightness and b) flatness.

After the model is presented above the marker, Fig. 3, the user can then start the measurement process or can use the yellow colour to track the system. In this case the user controls how fast the linear motion goes, or where it stops. Using "Change Material", different samples can be selected and entering the e-mail address the user will get the results by pressing the button "Send Mail".

\section{EXPLORATORY STUDY}

In order to evaluate the potential of such educational or training tools an exploratory study was performed, aiming to characterize a) the involvement of students using the application and its use as an online available experiment and b) how students perceive their experience and their learning through the use of an AR application.

\section{A. The sample}

The proof of concept of the straightness AR system was evaluated using a sample of 23 students of the Mechanical Engineering Integrated Master at the Faculty of Engineering of University of Porto.

\section{B. Implementation}

The students were told about the elementary concept of augmented reality followed by the explanation and demonstration of the AR straightness application. For fifteen minutes they were allowed to explore the AR tool. At the end they filled a survey in order to evaluate the AR tool usability in what concerns its effectiveness, satisfaction and achievement of specific goals. The number of questions related with effectiveness, satisfaction and goal achievement were balanced and the survey was short in order to guaranty the students' collaboration.

\section{Results}

Table 1 shows the results of the students' survey.

TABLE I.

SURVEY RESULTS (\%)

\begin{tabular}{|c|c|c|c|c|c|c|}
\hline Questions & TD & $\mathrm{D}$ & SD & SA & A & TA \\
\hline \multicolumn{7}{|l|}{ Effectiveness } \\
\hline 1 - Is the AR application intuitive? & & & 4 & 9 & 52 & 35 \\
\hline $\begin{array}{l}2 \text { - Does the application convey } \\
\text { the concept of AR? }\end{array}$ & & & & 4 & 70 & 26 \\
\hline \multicolumn{7}{|l|}{ Satisfaction } \\
\hline $\begin{array}{l}3 \text { - Is the application user- } \\
\text { friendly? }\end{array}$ & & & 4 & 9 & 70 & 17 \\
\hline 4 - Does it offer nice interactivity? & & & 4 & 9 & 61 & 26 \\
\hline \multicolumn{7}{|l|}{ Achieving specific goals } \\
\hline $\begin{array}{l}5 \text { - Is the application clear for the } \\
\text { straightness concept? }\end{array}$ & & & 4 & 22 & 57 & 17 \\
\hline
\end{tabular}
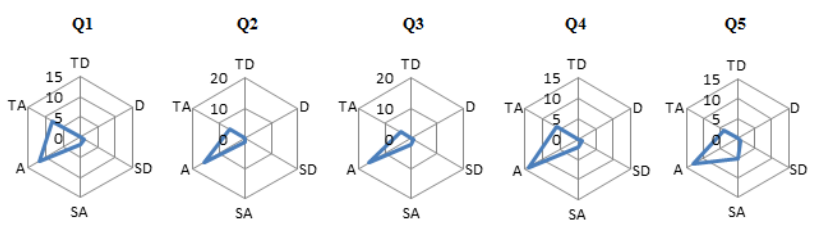

Figure 4. Survey results.

Six satisfaction levels (from "totally disagree" to "totally agree", identified as notes in Table I) were proposed in order to avoid centered answers.

Fig. 4 shows Table 1 results in a graphical way.

\section{FINAL COMMENTS}

The present AR application has been conceived to improve mechanical engineering students understanding and involvement in the straightness and flatness concepts.

The concept proof has been validated by the survey results. In the future these systems will be evaluated in industrial training environment. These applications will be improved by additional virtual guided interactive procedures for numerical evaluation of those geometrical characteristics.

\section{ACKNOWLEDGMENT}

The present work is due to the development and activities in online experimentation within the project Experiment@Portugal2102 funded by Calouste Gulbenkian Foundation and in agreement with other tasks related with the Internet of Things within PEstOE/EME/LA0022/2013, FCT.

\section{REFERENCES}

[1] J.F. Morie, "Virtual reality immersion and the unforgettable experience", Procs. of Electronica Imaging Stereoscopic Displays and Virtual Reality Systems", XIII, 2005.

[2] J.D. Meadows, Geometric Dimensioning and Tolerancing Handbook: Applications, Analysis \& Measurement, per ASME Y14.52009.

[3] Z. Jun-feng, W. Yan-yan, "Virtual Instrument of Measuring Machine Tool Guide Way Straightness Based on EST", Third International Conference on Information and Computing, Wuxi, China, 2010.

[4] T. Parisi, WebGL: Up and Running, Building 3D Graphics for the Web, O'Reilly, 2012.

\section{AUTHORS}

M. T. Restivo and M. F. Chouzal are with IDMECFEUP, Faculdade de Engenharia, Univ. Porto, Rua Dr. Roberto Frias, 4200-465 Porto, Portugal (trestivo@fe.up.pt, fchouzal@fe.up.pt).

J. Rodrigues is with Faculdade de Engenharia, Univ. Porto, Rua Dr. Roberto Frias, 4200-465 Porto, Portugal jose.c.rodrigues@gmail.com,

P. Menezes is with Instituto de Sistemas e Robótica, Univ. de Coimbra, Coimbra, Portugal (paulo@isr.uc.pt).

J. Almacinha is with the Mechanical Department at the Faculdade de Engenharia, Univ. Porto, Rua Dr. Roberto Frias, 4200-465 Porto, Portugal (jasa@fe.up.pt).

This work was supported in part by Fundação Calouste Gulbenkian. This article is an extended and modified version of a paper presented at the International Conference exp.at'13, held 18-20 September 2013, in Coimbra, Portugal. Submitted 18 November 2013. Published as resubmitted by the authors 04 December 2013. 